\title{
Modification of Recursive Kalman Filter Algorithm for Adaptive Prediction of Cyber Resilience for Industrial Systems
}

\author{
D. S. Lavrova* and D. P. Zegzhda ${ }^{\dagger}$ \\ Peter the Great St.Petersburg Polytechnic University, \\ 29 Politechnicheskaya Str, 195251 Saint-Petersburg, RUSSIA
}

(Received 14 July, 2020)

\begin{abstract}
This paper describes an approach to modification of the recursive Kalman filter algorithm to obtain adaptive prediction of time series from industrial systems. To ensure cyber resilience of modern industrial systems, it is necessary to detect anomalies in their work at an early stage. For this, data from industrial systems are presented as time series, and an adaptive prediction model combined with machine learning classification algorithm applies to identify anomalies. The effectiveness of the proposed approach is confirmed experimentally.
\end{abstract}

AMS Subject Classification: 00A06, 05C05, 05C20

Keywords: cyber resilience, time series, Kalman filter, anomaly prediction, anomaly detection

DOI: https://doi.org/10.33581/1561-4085-2020-23-3-270-279

\section{Introduction}

Digital transformation of the technological structure has led to the development of modern production technologies that integrate physical and information processes, as well as ensure independent functioning of industrial systems [1, 2]. Digitalization of industrial infrastructure has opened up great opportunities for implementation of computer attacks, the vector of which has been shifted towards unauthorized gaining the ability to control the system and violating the correctness of its functioning $[3,4]$.

The advent of cyberphysical systems has expanded the spectrum of cyberthreats $[5,6]$ : an increase in the number of cyberattacks, appearance of specific attacks on modern networks - wireless sensor networks and softwaredefined, destructive impact using malicious software, in particular, on components running under the Android operating system [7, 8].

The prevention of computer attacks on industrial systems, which allows to prevent irreversible physical processes from getting out of control, is a complicated technical issue due to the

\footnotetext{
${ }^{*}$ E-mail: lavrova@ibks.spbstu.ru

${ }^{\dagger}$ E-mail: dmitry@ibks.spbstu.ru
}

growing number of new types of computer attacks and the limited time to counter attacks.

To ensure cyber resilience, it is necessary to timely detect attacks on the industrial system and respond to them promptly so that the attacker does not have time to reach the goal. In such conditions, it is necessary to carry out early detection of computer attacks. Monitoring and analysis of data from all components of industrial systems will reveal anomalies in the system, which are often signs of computer attacks. In this regard, it is necessary to analyze data from industrial systems, their dynamics and characteristics. For this, it is proposed to use the presentation of data in the form of time series. Time series is a universal model which allows to submit data of any nature.

Application of adaptive prediction methods and models to time series will allow predicting future values of the series, given the changing external conditions. The recursive Kalman filter algorithm was chosen as such a model. However, this algorithm is mathematically complex, requires a priori knowledge of the system and knowledge of a large number of dependencies between system components. The process of setting up a Kalman filter for a specific system is time-consuming. This work is aimed at ensuring the universalization of this algorithm and making 
it applicable to any types of industrial systems to predict and maintain cyber-resilience.

\section{Related Works}

The issue of detecting computer attacks, especially on industrial systems, has been the subject of a large number of publications, for example, papers [9-11]. So, in [10], the authors propose an approach to detecting network attacks using a combination of detectors. Detectors are divided into groups, each of which is trained using a subsample of data from the initial training set. To obtain the final decision from a set of classifiers, a hybrid rule is applied based on the majority voting rule and the max-wins voting rule. It should be noted that testing was carried out using the 1998 DARPA test dataset, which does not fully reflect the modern network infrastructure of the substation, since the networks used and the data circulating in them have undergone significant changes. In this regard, this method may require some refinement when integrating it with the industrial system infrastructure.

Some studies focus on detecting specific types of attacks. The most common are studies that aim to identify Denial-of-Service (DoS) attacks. In [11], the authors propose a method for selecting network traffic indicators that most accurately reflect the difference between the normal functioning of the system and the system when a distributed denial of service (DDoS) attack is performed on it. To reduce computational complexity, the authors used two types of indicators: indicators describing the behavior of a particular packet (size, interval between sending a given packet and its predecessor, protocol), and indicators describing the general behavior of traffic (bandwidth, power of multiple recipient IP addresses, frequency sending to a specific IP address). The obtained indicators in combination with machine learning algorithms are used to detect attacks on routers or other intermediate network devices. The use of approaches based on machine learning can really significantly increase the accuracy of classification when detecting anomalies, but the paper does not say how much the training sample should be and how much time training should take. Also, this approach is focused specifically on the characteristics of network traffic, while the values of sensors and similar smart devices are also important indicators.

The development of methods and means of predictive analytics and predictive modeling currently goes more along the path of early diagnosis of malfunctions in industrial systems, as this will significantly save financial resources on repair and restoration work.

In the problem of prevention of computer attacks on industrial systems, prediction trends in system behavior is important. Detection of unwanted trends will signal the localization of security problems. Globally, among methods for predicting the state and behavior of a system, methods can be distinguished based on prediction models that operate on time series. The most common such models include:

1. regression models;

2. autoregressive models;

3. exponential smoothing models;

4. neural network models.

However, all these models have significant drawbacks for solving the problem of predicting the values of indicators of information infrastructure of the substation.

So, for regression models, the process of determining the model parameters is rather laborious. If the model is linear, then it is poorly adaptable and cannot model non-linear processes that are quite common in real systems. The authors of [12] propose using the method based on the regression model as a means of detecting anomalies in the readings of sensors of cyberphysical systems. The readings of each sensor are predicted as a function of the readings of other sensors. The interaction between the defense and the attacker is simulated as a Stackelberg model. During the simulation, the protection means determines a threshold value for 
each sensor, the transition beyond the boundaries of which signals an anomaly. This approach covers only those types of computer attacks that affect the performance of end devices. That is, the attacks carried out by the internal intruder and the attacks made on the internal elements of the network will go unnoticed if they do not affect the readings of the end devices. This approach will not be effective in the implementation of targeted attacks, the purpose of which is not to incapacitate the system, but to change the parameters of its functioning, remaining in the acceptable intervals of the values of the system devices.

The disadvantages of autoregressive models include a large number of model parameters, the identification of which is ambiguous and laborintensive. Like regression models, autoregressive models are characterized by low adaptability and inability to model nonlinear processes.

Exponential smoothing models are focused on long-term prediction, which is not suitable for substations, since short-term prediction is more important to prevent computer attacks. It should also be noted the lack of flexibility of such models to describe complex physical processes implemented by industrial systems.

Neural network models effectively cope with nonlinear processes, as studies show [1316]. Such models are adaptive and scalable, however, modeling is not transparent, therefore, errors may occur during training. Important disadvantages are also the complexity of the choice of architecture of neural networks, high requirements for the consistency of the training sample and the resource intensity of the learning process.

A promising approach to predicting the state of industrial system components is the use of the Kalman filter - a recursive filter that can adjust its state based on the received data. Mostly the Kalman filter is used in radiolocation problems, however, there are some examples of its use in cybersecurity [17-22]. In the source [21], the authors use the Kalman filter to predict the values of network parameters at strategically important points of the network infrastructure, which allows predicting possible network congestion. This task is not directly related to information security, but this approach will be able to catch overloads caused by the implementation of denial of service attacks on the network. The authors of the source [22] use the Kalman filter to detect False Data Injection Attacks, and it shows good results, but it is not clear why the authors do not extend this method to predict other types of attacks. Moreover, the sources considered do not indicate that the Kalman filter is only able to predict, but not determine which of the predicted values are abnormal. The Kalman filter is used only as a means of obtaining future values of the studied parameters, while its application can be expanded.

\section{Formalization of cyber- resilience prediction issue}

The considered object is an industrial system with a target function. Targer function of the industrial system is a set of physical processes, each of which corresponds to a set of functions $F_{i}$, performed by the components of the information part of industrial system: $F=\left\{F_{1}, F_{2}, \ldots, F_{n}\right\}, \forall F_{i}=\left\{f_{1}^{i}, f_{2}^{i}, \ldots, f_{k}^{i}\right\}$, with a given order relation. Each process is characterized by:

- data exchange between the components of the information component of the industrial system;

- the range of permissible values of the parameters of the information component of the industrial system, ensuring the correct course of the process.

The first step is to build a model of the functioning of an industrial system and show its completeness. The model should describe the objective function and the relationship between the functions $f_{k}, \ldots, f_{m} \in F_{i}$.

The model should describe the consequences of various types of computer attacks Z. Each cyber attack $Z_{i}$ affects a target function: $\forall i$ $f\left(Z_{i}, G\right): F \rightarrow F^{\prime}, F^{\prime} \neq F$ and realized in time time $\left(Z_{i}\right)$. 
The second step is to find the operator of early detection of attacks on the system. Attack detection operator Detect should be invariant to the type of cyber attack: $\forall i \exists \operatorname{Detect}\left(Z_{i}, G\right): \tilde{f}=$ $\left(\tilde{f}_{n}^{i}, \tilde{f}_{k}^{j}, \ldots\right)$ and detect anomalies in less time than the spread of the attack : time $\left(\right.$ Detect $\left.\left(Z_{i}, G\right)\right)<$ time $\left(Z_{i}\right)$.

To prevent computer attacks and to maintain cyber resilience, it is necessary to predict the behavior of system components, while the prediction must be adaptive, which means the ability of the prediction method to adjust its algorithm of operation depending on the input data.

\section{Modification of recursive Kalman filter algorithm}

The purpose of modifying Kalman filter algorithm was to detect computer attacks on industrial systems using a method that would allow detection of attacks at an early stage. This will enable the system security administrator to take measures to counteract the attack. The Kalman filter is an effective algorithm for obtaining an operational forecast one value ahead. However, in its classic form, the Kalman filter algorithm requires detailed consideration of each system under investigation and laborious calculation of parameters corresponding to a particular system. This process is extremely laborious and, moreover, it requires a large amount of a priori knowledge of the system, which is not always possible for industrial systems. Thus, the purpose of Kalman filter algorithm modification is its universalization for application to any type of industrial systems, without individual adjustment of algorithm parameters.

The essence of Kalman filter algorithm modification was to represent parameters from industrial systems as a body moving along the ordinate axis. Further, this model of body motion is used to set fixed parameters and matrices of the Kalman filter algorithm.

The Kalman filter implements two processes:

1. prediction - at this stage, the state of the system under study is predicted at the next time moment based on the filter readings obtained at the previous time moment;

2. correction - at this stage, the Kalman filter prediction is updated using the obtained real readings about the state of the system.

The operation scheme of the Kalman filter algorithm is shown in Figure 1.

Block 1 presents the initial estimated values of the predicted parameters $Y_{0}$ and $P_{0}$, where $Y_{0}$ is a vector of estimation of the initial value of the predicted parameter, $P_{0}$ is a covariance matrix of estimation errors at the initial step. Block 2 contains the values of the same parameters as block 1 , only at $k-1$ step.

Block 3 presents equations for estimating the current value of the predicted parameter based on the previous. $U_{k}$ is a vector of control action, the components of which characterize the elements of external influence on the system or system component. This vector is an optional element of the model, however, in the case of industrial systems, for which computer attacks are often implemented, its use is advisable. An $n \times n$ matrix A relates the state of the component in the previous time step to the state of the component in the current step. Matrix B is the control action matrix associated with the control action vector $U_{k}$, usually has a size $m \times n$. Matrix $W_{k}$ is a noise matrix. $W_{k}$ is a random process with a normal distribution with zero expectation and a covariance matrix $Q_{k}$, describing the random nature of the evolution of a system or a process:

$$
W_{k}=N\left(0, Q_{k}\right)
$$

Block 4 describes the process of calculating the Kalman gain $K$, where $H$ is the auxiliary matrix for reducing the Kalman gain to the matrix of the required dimension, $R$ is the measurement error. The Kalman gain $K$ is the most important parameter of the model; it represents the ratio between the errors of estimates constructed using the physical model of the system and the errors of measurements. In a simplified form, the formula for calculating the 


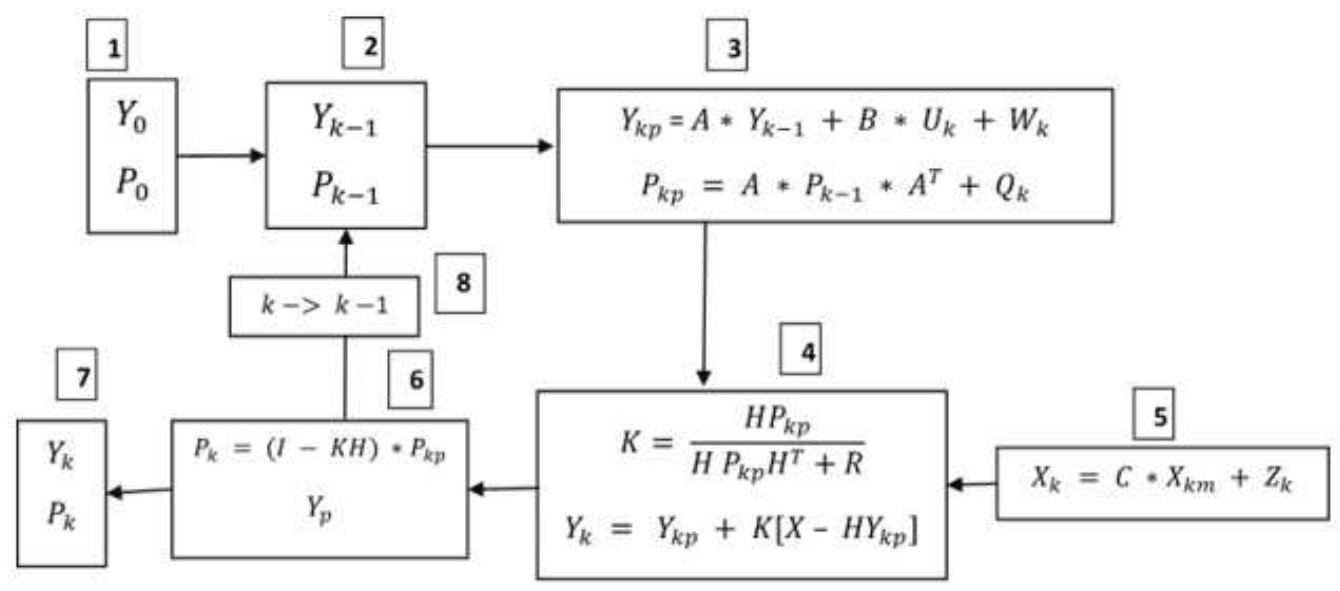

FIG. 1: Kalman filter algorithm.

value of this parameter is as follows:

$$
K=\frac{E_{\text {est }}}{E_{\text {est }}+E_{\text {meas }}}
$$

where $E_{e} s t$ is an evaluation error, $E_{m}$ eas is a measurement error.

The Kalman gain takes values from 0 to 1. If the Kalman gain $K$ is close to 1 , this means that the measurement error is small. If the value of this parameter is close to 0 , then the estimation error is large. Thus, the Kalman gain is an indicator of the degree of trust in the estimates obtained using the physical model of the system and the measurements obtained from the system components.

In block 5, data from the industrial system components are used; to obtain the value at the $k$ step, the matrix $C$ is used - a measurement matrix that relates the true values of the system state and the vector of measurements made, as well as the matrix $Z_{k}$, containing the values of the noise component. $Z_{k}$ is a normally distributed random variable with zero expectation and a covariance matrix $R_{k}$ :

$$
Z_{k}=N\left(0, R_{k}\right)
$$

In block 6 , the process of calculating the final filter result is described. Block 7 contains the values $Y_{k}$ and $P_{k}$, block 8 describes the repetition of the algorithm a given number of times under the assumption that the values calculated for step $\mathrm{k}$ will become the state for step $k-1$.
It should be noted that the composition of the vector $Y_{k}$ depends on the type of data being studied. For example, the elements of this vector can be estimates of the values at time $k$ of one-dimensional time series that are part of the multidimensional series under study. The vector $Y_{k}$ usually has a length $n$, where $n$ is the size of a multidimensional time series.

The "response" of the Kalman filter is a certain average value between the obtained estimates and the actual readings of the system components. These values are summed up with weights $(1-K)$ (weight of the system state assessment) and $K$ (weight of measurements).

Thus, if the measurement errors are very large and the Kalman gain tends to 0 , then it is believed that the calculated estimates more accurately reflect the real picture of the process and their weight will be greater than the weight of the sensor readings. If the measurement errors are small, then the Kalman gain tends to 1, and the degree of confidence in the sensor readings is higher than the estimates, since it is believed that they are more accurate.

Thus, the modified Kalman filter algorithm includes the following steps:

1. The initial indications of the system component are taken as initial estimates.

2. Then these initial values are taken as the component at the previous time. 
3. The component readings in the current period are estimated based on the previous one. At this step, it is necessary to update the covariance matrix based on the previous covariance matrix using matrix $A$. At this step, the first stage completed by the filter ends, namely, the prediction stage. In the following steps, the algorithm adjusts the resulting estimate.

4. The Kalman gain $K$ is calculated. Here $R$ is the measurement error (readings of the system component). Sometimes $R$ is constructed based on the technical characteristics of the components (measurement error, which is usually indicated in the accompanying documents), if such information is available.

5. Then it is necessary to obtain the measurements from the system components with the characteristics calculated on them. In the algorithm diagram, this data is also represented by the matrix $C$ and the noise matrix $Z$.

6. Based on the Kalman gain obtained, the previously obtained estimate and the available measurement from the component, the final filter response is calculated.

7. The received response of the Kalman filter is taken as indicators of the state of the system component at time $k$.

8. It is assumed that the state of a component obtained earlier using the algorithm at time $k$ becomes its state at time $k-1$, and the steps of the algorithm are repeated as many times as necessary [23].

\section{Industrial data representation model for Kalman filter}

At the first stage of the implementation of the algorithm, it is necessary to determine the composition of the above matrices and vectors. To construct these matrices, information is needed on the physical model of the system under study and a diagram reflecting the physical relationships between its parts and components. However, for PS this information is not always available.

To solve this problem, it is proposed to present the testimony of each component of the PS ("field"level) as a chaotic trajectory of motion of a body. The body at each moment in time has a different speed: it either accelerates or slows down, changing its position every second.

It is assumed that the PS components do not measure physical quantities, but the position of certain bodies in one-dimensional space. Moreover, the position of the bodies on the ordinate axis in the Cartesian system is considered. Each time series of indications of a particular PS component corresponds to the trajectory of movement of each individual body. This body is characterized by the following parameters:

- $Y$ - body position on the $\mathrm{Y}$ axis;

- $V$ - body speed;

- $a$ - acceleration.

Body speed is calculated as:

$$
V_{y}=\frac{s_{y}}{t}
$$

where $S$ is the distance traveled by the body along the axis $Y$.

The distance traveled by the body is calculated as the difference in the coordinates of the position of the body on the $\mathrm{Y}$ axis at the current and previous time. Since it was previously assumed that the measurement of the position of the body is performed every second, then $\Delta t=1$. Thus, the speed of the body is equal to the difference in coordinates between the current and previous time.

Every second, the body has a different speed, so acceleration must be taken into account when building the model. In this case, acceleration is considered as the difference in speeds at the current and previous time.

The motion of the body (calculating the coordinate of the body) in one-dimensional space 
is usually described by the equation:

$$
y_{t}=y_{t-1}+V * t+\frac{a}{2} * t^{2}
$$

Assessment of body position, according to the Kalman filter algorithm, is performed according to the following equation:

$$
y_{k}=A * y_{k-1}+B * U_{k}+W_{k}
$$

where $A$ is a process evolution matrix, $y_{k-1}$ is an object state vector at the moment $(k-1), U_{k}$ is a control vector, $B$ is a control matrix, which is applied to the control vector $U_{k}, W_{k}$ is noise.

In this situation, the state vector of the object at time $k-1$ consists of the coordinate of the object on the $\mathrm{Y}$ axis at time $k-1$ and the speed of the object at $k-1$ time moment:

$$
Y_{k-1}=\left[\begin{array}{l}
y_{k-1} \\
V_{k-1}
\end{array}\right]
$$

The control vector contains acceleration. Since the speed of the object changes under the influence of acceleration, this parameter will be determined as a controlling external parameter. Thus, the vector $U_{k}$ is as follows:

$$
U_{k}=\left[a_{k}\right] .
$$

When a moving body is considered, the matrices $A$ and $B$ have the standard form:

$$
A=\left[\begin{array}{cc}
1 & \Delta t \\
0 & 1
\end{array}\right], \quad B=\left[\begin{array}{c}
\frac{\Delta t^{2}}{2} \\
\Delta t
\end{array}\right] .
$$

The matrix $W$ in this case is the zero matrix. This type of matrix is explained by the physics of the process. If we substitute all the matrices and vectors obtained earlier, we get the following result:

$$
\begin{aligned}
Y_{k} & =\left[\begin{array}{cc}
1 & \Delta t \\
0 & 1
\end{array}\right] *\left[\begin{array}{c}
y_{k-1} \\
V_{k-1}
\end{array}\right]+\left[\begin{array}{c}
\frac{\Delta t^{2}}{2} \\
\Delta t
\end{array}\right] *\left[a_{k}\right]+0 \\
& =\left[\begin{array}{c}
y_{k-1}+V_{k-1} * \Delta t+\frac{\Delta t^{2}}{2} * a_{k} \\
V_{k-1}+a_{k} * \Delta t
\end{array}\right]
\end{aligned}
$$

Thus, a new state of the body was obtained at time $k$. The first element of the resulting vector is the coordinate of the body on the axis at time $k$. As you can see, the resulting formula for the coordinate of the body is fully consistent with the equation that is used to study the motion of the body. The second element of the obtained state vector is responsible for the velocity value at a new moment in time. The speed of a body at time $k$ is equal to the sum of the speed of this body at time $k-1$ and its acceleration at time $k$, which also fully complies with physical laws.

Elements of the matrix $Q$ were selected experimentally. The process took place in the following way: random numbers (generated by a random number sensor), whose values were within the range of 0 to 1 , were chosen as elements of matrix $Q$. In the course of the experiment it was found that if you choose numbers within the range from 0 to 1 , the result does not change much, regardless of what values some or other matrix elements have taken. Then other numerical parameters were used to generate matrix $Q$ and the results were worse. Therefore the decision was made to take random numbers within the range of 0 to 1 as matrix $Q$ elements.The matrix $H$ is an auxiliary matrix that is used to reduce the Kalman gain to a matrix of the desired dimension (in this case, $2 \times 2$ ). Usually, in such a problem, $H$ is the identity matrix.

\section{Experimental studies}

To automatically classify the values predicted by the modified Kalman filter algorithm, the Random Forest machine learning method was used. He has proven himself in the tasks of classification, also it is resistant to re-training.

Experimental studies to evaluate the effectiveness of the developed method were carried out on an industrial automatic water purification system. Work with this system was performed on the basis of the SWaT data set provided by the University of Singapore [24]. The system is a small copy of the water treatment 
plant, which goes through two stages of filtration. The system imitates the operation of large modern water treatment plants, such as those used in urban services that supply the population with water.

The water purification process in this system includes six stages. Monitoring the progress of each stage and monitoring processes is carried out using a large set of sensors. A detailed description of each sensor is presented in the source [24]. The data set includes indicators of both the normal operation of the system and the operation of the system in conditions of computer attacks. A total of 51 system sensors are recorded recorded every second.

The results of the Kalman filter on the example of several series from the SWaT data set are presented in Figures 2 and 3, real data are shown by a straight line, and predicted by a dashed line. The data in Figures 1 and 2 are obtained as follows. The data from the automatic water treatment system containing both normal values of the system components and abnormal (caused by attacks) values were used. These data were divided into test and training samples. Figures 1 and 2 show the results of the Kalman filter-based forecast for the training sample. You can see from the figures that all bursts and drops were correctly predicted.

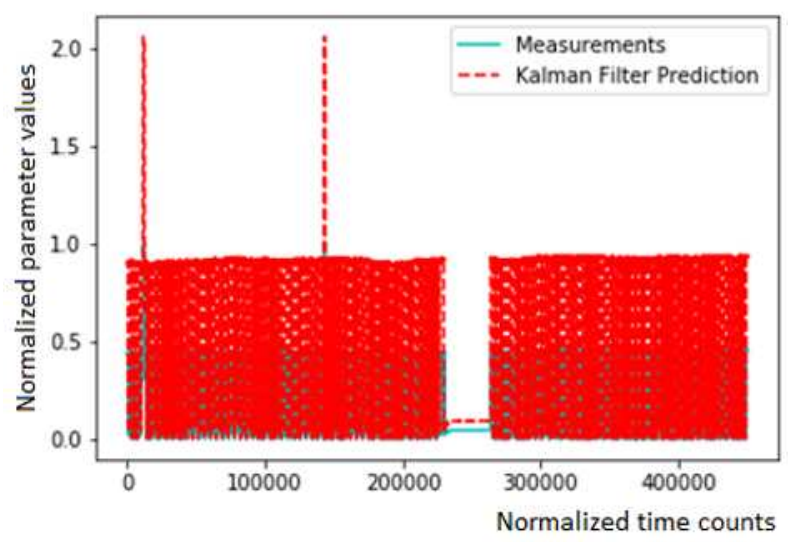

FIG. 2. (color online) The results of the Kalman filter (1).

From the graphs presented in Figures 1, 2, it can be seen that the Kalman filter predicts the

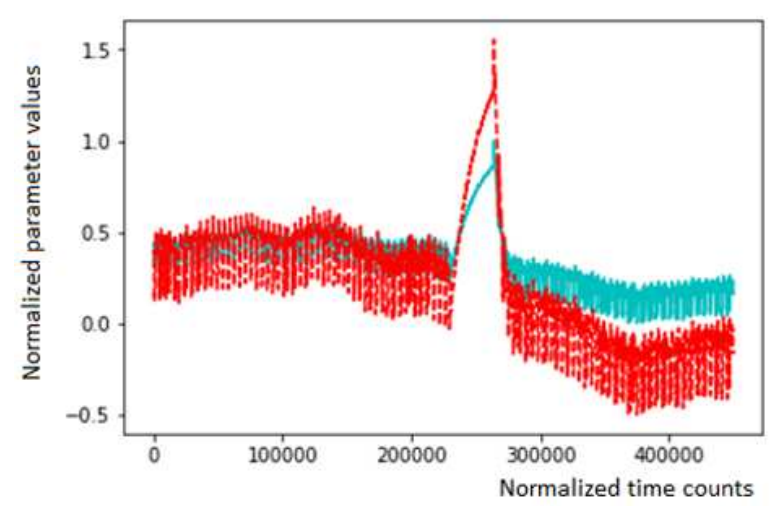

FIG. 3. (color online) The results of the Kalman filter (2).

Table 1. Values of the Random Forest performance indicators.

\begin{tabular}{|c|c|}
\hline Quality metric name & Quality metric value \\
\hline Accuracy & 0.96 \\
\hline Precision & 0.96 \\
\hline Recall & 0.94 \\
\hline
\end{tabular}

dynamics of the sensor readings quite well both at the time of normal operation of the system and at the time of the attack.

The calculation time of the Kalman filter readings for one time series from the considered data set (the length of the series is 449917 observations) was approximately 41.79 seconds. The time the model spends calculating the forecast one observation ahead was approximately 0.0001 seconds.

The division of data into training and test samples was made in the following way. The predictions obtained using the filter were divided into training and test samples in the ratio of $70 \%$ and $30 \%$, respectively. At the same time, the values for each class were selected randomly rather than in order. The main condition for dividing data into two samples was that objects of both classes - both normal values and anomalous ones - must necessarily fall into each sample.

The values of the Random Forest performance indicators on the test set are presented in Table 1. The forest contains 30 trees, the depth of each of which does not exceed 7 levels: 
Learning the algorithm took about 6 seconds, while the algorithm on the test sample took about 1 second.

\section{Conclusion}

Thus, we can conclude that the developed method of detecting computer attacks based on the modified Kalman filter algorithm and machine learning is highly efficient and is characterized by high speed.

The efficiency of the proposed method is justified by the results of correct classification of the predicted values from the water treatment system into normal and abnormal ones. This is emphasized by high values of accuracy, precision and recall metrics (0.94-0.96). The high speed of the method is justified by the fact that the calculation time of the Kalman filter readings for one time series is approximately 41.79 seconds, and to predict the next value requires about 0.0001 seconds. Taken together, this time is less than the spread time of the attacks implemented over the water treatment system under consideration. Therefore, for such systems, this method allows fixing an attack at an early stage, leaving time to react to it.

It should also be noted that the part of the method that is not related to machine learning requires a small amount of memory, since the Kalman filter does not require a large amount of data about previous readings of a system component, it needs only one past value.
Benefits of the proposed method for detection cyber attacks, based on Kalman filter modification and machine learning:

- prediction horizon - from a few seconds to a few months;

- $94-99 \%$ prediction accuracy;

- high speed of prediction - less than 0.1 second;

- adaptability to changes in time series, ability to catch the trend;

- universality - applicability to data of any nature (network traffic, financial data, sensor data etc.).

Using this modified algorithm, the values of indicators of industrial systems could be predicted. It also could be applied for predicting the cyber resilience value of the system. Cyber resilience can be estimated using various metrics, however it is closely related to the parameters of the system [25]. Thus, the proposed approach is effective for use in the field of cybersecurity.

\section{Acknowledgements}

The study was carried out as part of a scholarship of the President of the Russian Federation to young scientists and graduate students SP-1932.2019.5.

\section{References}

[1] M. N. Sadiku, Y. Wang, S. Cui, and S. M. Musa, European Scientific Journal 13, 52 (2017).

[2] S. K. Khaitan and J. D. McCalley, IEEE Systems Journal 9, 350 (2014).

[3] G. N. Ericsson, IEEE Transactions on Power Delivery 25, 1501 (2010)

[4] M. A. Poltavtseva, in 2019 International Conference on Engineering Technologies and Computer Science (EnT) (IEEE, 2019), pp. 25-
29.

[5] Y. S. Vasilв $丂^{\mathrm{TM}}$ ev, D. P. Zegzhda, and M. A. Poltavtseva, Automatic Control and Computer Sciences 52, 1090 (2018).

[6] S. I. Shterenberg and M. A. Poltavtseva, Automatic Control and Computer Sciences 52, 945 (2018).

[7] M. O. Kalinin and E. Y. Pavlenko, Automatic Control and Computer Sciences 49, 673 (2015). 
[8] P. Zegzhda, D. Zegzhda, E. Pavlenko, and A. Dremov, in Proceedings of the 10th International Conference on Security of Information and Networks (2017), pp. 280283.

[9] E. Y. Pavlenko, A. Yarmak, and D. A. Moskvin, Automatic Control and Computer Sciences 51, 829 (2017).

[10] A. Branitskiy and I. Kotenko, in AI in Cybersecurity (Springer, 2019), pp. 115-149.

[11] R. Doshi, N. Apthorpe, and N. Feamster, in 2018 IEEE Security and Privacy Workshops (SPW) (IEEE, 2018), pp. 29-35.

[12] A. Ghafouri, Y. Vorobeychik, and X. Koutsoukos, arXiv preprint arXiv:1804.11022 (2018).

[13] K. Hundman, V. Constantinou, C. Laporte, I. Colwell, and T. Soderstrom, in Proceedings of the 24th ACM SIGKDD international conference on knowledge discovery \& data mining (2018), pp. 387-395.

[14] P. Filonov, A. Lavrentyev, and A. Vorontsov, arXiv preprint arXiv:1612.06676 (2016).

[15] A. Nanduri and L. Sherry, in 2016 Integrated Communications Navigation and Surveillance (ICNS) (Ieee, 2016), pp. 5C2-1.

[16] S. Yi, J. Ju, M.-K. Yoon, and J. Choi, arXiv preprint arXiv:1703.09938 (2017).

[17] C.-Z. Bai and V. Gupta, in 2014 American control conference (IEEE, 2014), pp. 3029-3034.

[18] K. Manandhar, X. Cao, F. Hu, and Y. Liu, IEEE transactions on control of network systems $\mathbf{1}, 370$ (2014).

[19] Z. Zhang, J. Wu, D. Yau, P. Cheng, and J. Chen, in 2018 ACM/IEEE 9th International Conference on Cyber-Physical Systems (ICCPS) (IEEE, 2018), pp. 345-346.

[20] A. Jain and N. Shivakumar, in 2008 40th North American Power Symposium (IEEE, 2008), pp. $1-8$.

[21] J. Haught, K. Hopkinson, N. Stuckey, M. Dop, and A. Stirling, in Proceedings of the 2010 Winter Simulation Conference (IEEE, 2010), pp. 2927-2934.

[22] Q. Yang, L. Chang, and W. Yu, Security and Communication Networks 9, 833 (2016).

[23] G. Welch, G. Bishop, et al., An introduction to the kalman filter (1995).

[24] J. Goh, S. Adepu, K. N. Junejo, and A. Mathur, in International Conference on Critical Information Infrastructures Security (Springer, 2016), pp. 88-99.

[25] D. P. Zegzhda and E. Y. Pavlenko, Automatic Control and Computer Sciences 52, 1150 (2018). 